\title{
Sufficient separability criteria and linear maps
}

\author{
Maciej Lewenstein, ${ }^{1,2, *}$ Remigiusz Augusiak, ${ }^{1,3}$ Dariusz Chruściński, ${ }^{4}$ Swapan Rana, ${ }^{1}$ and Jan Samsonowicz ${ }^{5}$ \\ ${ }^{1}$ ICFO - Institut de Ciències Fotòniques, The Barcelona Institute of Science and Technology, 08860 Castelldefels, Spain \\ ${ }^{2}$ ICREA - Institució Catalana de Recerca i Estudis Avançats, Lluis Companys 23, 08010 Barcelona, Spain \\ ${ }^{3}$ Center for Theoretical Physics, Polish Academy of Sciences, Al. Lotników 32/46, 02-668 Warsaw, Poland \\ ${ }^{4}$ Institute of Physics, Faculty of Physics, Astronomy and Informatics, \\ Nicolaus Copernicus University, Grudziadzka 5/7, 87-100 Torun, Poland \\ ${ }^{5}$ Faculty of Mathematics and Information Science, Warsaw University of Technology, Pl. Politechniki 1, 00-61 Warszawa, Poland
}

\begin{abstract}
We study families of positive and completely positive maps acting on a bipartite system $\mathbb{C}^{M} \otimes \mathbb{C}^{N}$ (with $M \leq N$ ). The maps have a property that when applied to any state (of a given entanglement class) they result in a separable state, or more generally a state of another certain entanglement class (e.g., Schmidt number $\leq k$ ). This allows us to derive useful families of sufficient separability criteria. Explicit examples of such criteria have been constructed for arbitrary $M, N$, with a special emphasis on $M=2$. Our results can be viewed as generalizations of the known facts that in the sufficiently close vicinity of the completely depolarized state (the normalized identity matrix), all states are separable (belong to "weakly" entangled classes). Alternatively, some of our results can be viewed as an entanglement classification for a certain family of states, corresponding to mixtures of the completely polarized state with pure state projectors, partially transposed and locally transformed pure state projectors.
\end{abstract}

PACS numbers: 03.65.Aa, 03.67.Hk

\section{INTRODUCTION}

Entanglement is a property of density operators identified with quantum mechanical states of a multipartite system. Such operators act on the corresponding Hilbert space, which has a fixed tensor structure, corresponding to different parties (Alice, Bob, Charlie,...). Notably, entanglement is the most fundamental resource for quantum information processing [1]. For this reasons characterization of entanglement and related quantum correlations is one of the most important tasks in quantum information science. Yet, it is a very challenging task, since even for bipartite systems the problem of determining if a given state is entangled or not is NP-hard [2] from the computational point of view. Although in low dimensions there exist efficient numerical codes checking entanglement using semi-definite programming [3], this problem becomes untractable when the dimension of the underlying tensor product Hilbert space grows. For this reason it is extremely important to develop and have at our disposal operational entanglement criteria that can be used in experiments.

So far, most of the known separability criteria are necessary (that is sufficient for detecting entanglement), starting with the prominent positive partial transpose criterion, proposed by Peres [4], and also proven to be sufficient for two-qubits and one qubit-qutrit by Horodeckis [5]. In fact transposition $T$ is a paradigmatic example of a positive map, i.e., a map that maps states onto states. Transposition is not, however, completely positive, i.e., its extensions by identity map to larger tensor spaces do not correspond to positive maps. This

\footnotetext{
* maciej.lewenstein@icfo.es
}

is actually a general fact, proven by Horodeckis (cf.[1]): any positive map acting exclusively on a space of Alice's (Bob's) operators, transforms a separable state into a positive definite state. Conversely, a state is separable if it remains positive definite under action of all positive map on Alice's (Bob's) side. Several examples of positive maps have been proposed and studied in the context of entanglement: cf. the celebrated reduction map [6, 7], Breuer-Hall map [8, 9], or Choi map [10]. Note that in contrast to the Breuer-Hall and Choi maps, the reduction map is decomposable, i.e., it does not allow to detect entanglement of states with positive partial transpose. Also, note that positive maps are not "physical" and cannot be realized directly in experiments; nevertheless, it is possible to realize them indirectly, using the, so called, structural approximations to positive maps [1115], or multi-copy entanglement witnesses [16, 17].

Another common approach to assure separability and detect entanglement is based on the celebrated entanglement witnesses (cf. [1, 18, 19]). These are observables, whose average value is non-negative on all separable states, whereas it is strictly negative on an entangled state. Witnesses detect some entangled states only, but their advantage is that they can be directly measured using local optimized measurements [20]. Generalizations of witnesses and maps to the non-linear witnesses and maps are possible, but rare and not so frequently used (cf. [1]).

Sufficient criteria of separability (i.e., necessary entnaglement criteria) are not so common. They appeared very early in the theory of entanglement in the studies of robustness of entanglement [21]. More importantly, sufficient sepaprability criteria turn out to be crucial in the studies of volume of separable states, which amounts to estimations of the size of the ball around the separable states that contains separable states only [22]. These 
ideas proved being important for the general aspects of quantum computing-indeed they allowed Braunstein et al. to demonstrate that the high temperature NMR "quantum computing" does not involve entanglement [23]. This important problem has been followed by many researchers-"classical" results were obtained for instance by Barnum and Gurvits [24]; Later, in the series of works by Szarek et al. [25, 26] powerful estimations of the volume of separable states were made for multipartite systems.

Here we present alternative and general approach to derive sufficient criteria for separability and more generally of belonging to a certain class of entanglement, quantum correlations etc. Alternatively, we will derive conditions when a state of a certain form (corresponding to a mixture of the completely polarized state with pure state projectors, partially transposed and locally transformed pure state projectors etc.) belongs to a certain entanglement/correlation class. Our approach is somewhat related to that of Barnum and Gurvits: we consider families of maps acting on global states of a bi- or multiparty system, having the property that when applied to a state (a state of a certain entanglement class) they produce a separable state (a state of another certain entanglement class).

The paper is organized as follows. In Section II we present our notation, definitions, and preliminary facts. In Section III we explain the general theorem, on which we then base our sufficient criteria, and sketch the previously known results - in particular we remind the reader the results of Vidal, Barnum and Gurvits. Main results for $2 \otimes N$ and $M \otimes N$ systems are presented in Sections IV and $\mathrm{V}$, respectively. We focus mainly on sufficient separability criteria, but also discuss some results that go beyond this paradigm. We close the paper with the conclusions and outlook in Section VI.

\section{PRELIMINARIES}

We consider states of a bipartite system of Alice and Bob, described by density operators $\rho$ acting on the Hilbert space $\mathcal{H}=\mathcal{H}_{A} \otimes \mathcal{H}_{B}=\mathbb{C}^{M} \otimes \mathbb{C}^{N}$ with $M \leq N$. The space of all bounded operators acting on it is denoted by $\mathcal{B}\left(\mathcal{H}_{A} \otimes \mathcal{H}_{B}\right)$, while the set of states, that is, operators from $\mathcal{B}\left(\mathcal{H}_{A} \otimes \mathcal{H}_{B}\right)$ obeying $\rho \geq 0, \rho=\rho^{\dagger}$ and $\operatorname{Tr}(\rho)=1$, as $R$ (also denoted as $\Sigma_{M}$, see below). Note that $R$ is convex and compact. However, the normalization condition of the states and, consequently, the compactness of $R$ will only be assumed if necessary. The transposed operator $\rho$ will be denoted as $\rho^{T}$, whereas partially transposed as $\rho^{T_{A}}\left(\rho^{T_{B}}\right)$. In the following we will consider also various classes of states such as:

- Separable states $\Sigma$, also denoted as $\Sigma_{1}$, i.e., states that admit the following decomposition

$$
\sigma=\sum_{k}^{K} p_{k}\left|\Psi_{k}\right\rangle_{1}\left\langle\left.\Psi_{k}\right|_{1},\right.
$$

where $\left|\Psi_{k}\right\rangle_{1}=\left|e_{k}\right\rangle \otimes\left|f_{k}\right\rangle$ are simple tensors, i.e., product also know as vectors with Schmidt rank 1 , i.e., having only one non-vanishing Schmidt coefficient [27].

- States with Schmidt number $n \leq M, \Sigma_{n}$, i.e., the states that admit the decomposition

$$
\sigma=\sum_{k}^{K} p_{k}\left|\Psi_{k}\right\rangle_{n}\left\langle\left.\Psi_{k}\right|_{n}\right.
$$

where $\left|\Psi_{k}\right\rangle_{n}=\sum_{l=1}^{n} \lambda_{l}\left|e_{k l}\right\rangle \otimes\left|f_{k l}\right\rangle$ are vectors with Schmidt rank $\leq n$, and at least one of them has Schmidt rank equal to $n[28,29]$.

- PPT-operators $W_{P P T}$, i.e., those that have a positive patrial transpose, $w^{T_{A}} \geq 0$; in particular, we will talk about PPT-states, $R_{P P T}$, i.e., $\rho \in R$ and $\rho \in W_{P P T}$, i.e., $\rho^{T_{A}} \geq 0$.

- Pre-witnesses of entangled states $W$, also denoted as $W_{1}$, i.e., observables $W=W^{\dagger}$ such that for every $w \in W$ and every $\sigma \in \Sigma$, we have $\operatorname{Tr}(w \sigma) \geq 0$. Note that the genuine witnesses additionally must not be positive definite, i.e., $w \notin R=\Sigma_{M}[1,18]$

- Pre-witnesses of entangled states of Schmidt number $n, W_{n}$, i.e., observables $W=W^{\dagger}$ such that for every $w \in W_{n}$ and every $\sigma_{n} \in \Sigma_{n}$, we have $\operatorname{Tr}\left(w_{n} \sigma_{n}\right) \geq$ 0 . Note that the genuine witnesses additionally must detect a state from $\Sigma_{n+1}$, there must exist a $\sigma_{n+1} \in \Sigma_{n+1}$, such that $\operatorname{Tr}\left(w_{n+1} \sigma_{n}\right)<0[28,29]$.

- Decomposable entanglement pre-witnesses, i.e., pre-witnesses that admit a decomposition $W=$ $P+Q^{T_{A}}$, where $P, Q \geq 0$. Witnesses that do not admit such decomposition are termed nondecomposable (cf. $[18,19])$.

Consequently, we will consider various classes of linear maps

$$
\Lambda: \mathcal{B}\left(\mathcal{H}_{A} \otimes \mathcal{H}_{B}\right) \rightarrow \mathcal{B}\left(\mathcal{H}_{A} \otimes \mathcal{H}_{B}\right)
$$

that preserve hermiticity. We will pay particular attention to those maps that map a certain subset of $S \subset$ $\mathcal{B}\left(\mathcal{H}_{A} \otimes \mathcal{H}_{B}\right)$ to another certain subset of $S^{\prime} \subset \mathcal{B}\left(\mathcal{H}_{A} \otimes \mathcal{H}_{B}\right)$, i.e., for every $w \in S$ we have $\Lambda(w) \in S^{\prime}$. We will typically consider families of maps $\Lambda_{\mathbf{p}}$, parameterized by a set of parameters $\mathbf{p}=(\alpha, \beta, \gamma, \ldots)$, and assume that the map is invertible for almost all values of the parameters. We will denote by $R(\rho), K(\rho)$, and $r(\rho)=\operatorname{dim} R(\rho)$ the range, the kernel and the rank of $\rho$, respectively. Complex conjugation will be denoted according to the "physical tradition" as *, so that the complex conjugate of a vector $|e\rangle$ is $\left|e^{*}\right\rangle$, while any perpendicular vector by $\left|e^{\perp}\right\rangle$. Because of the reasons that will become clear below we will mostly work with even $M$ and $N$. In such case it is straightforward to implement in an easy way, the so called, BreuerHall unitary operators $V, V V^{\dagger}=\mathbb{1}$, such that for all vectors $|e\rangle$,

$$
V\left|e^{*}\right\rangle=\left|e^{\perp}\right\rangle
$$




\section{GENERAL THEOREMS}

Our approach is mainly based on the following simple observation.

Theorem 1. Let $S, S^{\prime}$ are convex and compact subsets of $\mathcal{B}\left(\mathcal{H}_{A} \otimes \mathcal{H}_{B}\right)$, and let $\Lambda_{\mathbf{p}}: S \rightarrow S^{\prime}$ be a family of maps, invert$i$ ible for almost all $\mathbf{p}$. Let $\mathcal{P}_{S S^{\prime}}$ denote a subset of the parameters set, and the maps have the property that for every $w \in S$ we have that $\Lambda_{\mathbf{p}}(w) \in S^{\prime}$, provided $\mathbf{p} \in \mathcal{P}_{S S^{\prime}}$. Then $\Lambda_{\mathbf{p}}^{-1}(\sigma) \in S$ $\Rightarrow \sigma \in S^{\prime}$.

Proof. Note that $\Lambda_{\mathbf{p}}\left[\Lambda_{\mathbf{p}}^{-1}(\sigma)\right]=\sigma$.

Now let $S$ be the set of all $M \otimes N$ states and $S^{\prime} \subset S$ be the separable states. This general theorem can be applied to derive sufficient separability criterion, provided the choice of $\Lambda_{p}$ is such that

i) we can easily check that $\Lambda_{\mathbf{p}}^{-1}(S) \subset S$,

ii) we can prove the assumption that $\Lambda_{\mathbf{p}}(S) \subset S^{\prime}$.

Clearly, the difficulty of deriving the sufficient separability criteria is hidden in the condition ii).

Below we provide some examples (of sufficient separability criteria) known from the literature, to illustrate concrete use of Theorem 1. Let us consider the simple reduction-type map $\Lambda_{\alpha}(\rho):=\operatorname{Tr}(\rho) \mathbb{1}+\alpha \rho$. For condition ii), we have to find the range of the parameter $\alpha$ such that $\Lambda_{\alpha}(\rho)$ is separable. Once this is done, $\Lambda_{\alpha}^{-1}(\rho) \geq 0$ would be a sufficient separability criterion. Separability of $\Lambda_{\alpha}(\rho)$ is well known and given by the following result.

Theorem 2. [21, 24] Let $\Lambda_{\alpha}(\rho)=\operatorname{Tr}(\rho) \mathbb{1}+\alpha \rho$ be the family of maps, and $-1 \leq \alpha \leq 2$. Then $\rho \geq 0 \Rightarrow \Lambda_{\alpha}(\rho)=: \sigma \in \Sigma$, i.e. $\sigma$ is separable.

Proof. It is enough to prove the theorem for pure states, $\rho=|\Psi\rangle\langle\Psi|$. For $M=2,|\Psi\rangle$ has maximally Schmidt rank 2 , and, without loosing generality, we can assume that $|\Psi\rangle=\lambda_{0}|0\rangle \otimes|0\rangle+\lambda_{1}|1\rangle \otimes|1\rangle$. It is then enough to check positivity and separability of $\Lambda(|\Psi\rangle\langle\Psi|)$ on a $2 \otimes 2$ space spanned by $|0\rangle,|1\rangle$ in both Alice's and Bob's spaces. PPT provides then necessary and sufficient condition [5], and we easily get that indeed $-1 \leq \alpha \leq 2$. The condition $\alpha \geq$ -1 actually follows already from a simpler requirement of positivity of $\Lambda_{\alpha}(\rho)$, as it appears in the definition of the reduction map [6, 7].

To prove the theorem for $M \geq 3$ we exploit the fact, proven in Ref. [21] (see also Ref. [14]) that the (unnormalized) mixture of $|\psi\rangle$ and the maximally mixed state, $\mathbb{1}+\alpha|\psi\rangle\langle\psi|$, is separable iff

$$
\alpha \leq \frac{1}{\lambda_{0} \lambda_{1}} .
$$

This implies that $\mathbb{1}+\alpha|\psi\rangle\langle\psi|$ is separable for any $|\psi\rangle$ if $\alpha \leq 2$.

This leads to the following separability criteria.
Theorem 3. (Sufficient separability criterion 1) If $\Lambda_{\alpha}^{-1}(\sigma):=[\sigma-\operatorname{Tr}(\sigma) \mathbb{1} /(2 N+\alpha)] / \alpha \geq 0$ then $\sigma \in \Sigma$, i.e. $\sigma$ is separable.

In particular, for the extreme values of $\alpha$, we get the following separability results.

Corrolary 4. If $\sigma-\operatorname{Tr}(\sigma) \mathbb{1} /(2 N+2) \geq 0$ then $\sigma \in \Sigma$, i.e. $\sigma$ is separable.

Corrolary 5. If $\operatorname{Tr}(\sigma) \mathbb{1} /(2 N-1)-\sigma \geq 0$ then $\sigma \in \Sigma$, i.e. $\sigma$ is separable.

It is amazing that these simple criteria are strong enough to detect some states outside the "separable ball" around identity [24] (see Section IV C for an explicit example and detailed discussion). Of course, not all states from the separable ball are detected. The reason for this weakness is that Barnum and Gurvits have estimated the radius of the separable ball using a stronger result, namely: if the operator norm of a Hermitian matrix $X$ is bounded by 1 , then $\mathbb{1}+X$ is separable. In contrast, our simple criteria depend on the spectrum, not on the norm, and are thereby independent. Nonetheless, we will see in the next sections that with other choices of $\Lambda_{\mathbf{p}}$ 's, we could derive further stronger sufficient separability criteria.

\section{MAIN RESULTS FOR $\mathbb{C}^{2} \otimes \mathbb{C}^{N}$}

\section{A. Reduction- and Breuer-Hall-like maps}

Let us start by presenting generalizations of Theorem 2 derived in a similar spirit as the generalization of the reduction map by the Breuer-Hall map [8,9]. Note that Breuer-Hall's construction does not work in the qubit case-Breuer-Hall's map identically vanishes for qubits. In our case when we consider maps acting on the composite space of Alice and Bob this restriction will not apply. In the two-dimensional space there exist (up to a phase) a single unitary, $\sigma_{2}$, with the property that for every $|e\rangle, \sigma_{2}\left|e^{*}\right\rangle=\left|e^{\perp}\right\rangle$. For simplicity we will consider the case when Bob's space has even dimension; in such a case it is easy to construct analogous Breuer-Hall unitary operator $V$, such that $V\left|f^{*}\right\rangle=\left|f^{\perp}\right\rangle$.

Below we will denote, for brevity:

$$
\begin{gathered}
\tilde{\rho}_{A}=\sigma_{2} \rho^{T_{A}} \sigma_{2}, \\
\tilde{\rho}_{B}=V \rho^{T_{B}} V^{\dagger},
\end{gathered}
$$

and

$$
\tilde{\rho}=\sigma_{2} V \rho^{T} V^{\dagger} \sigma_{2} .
$$

We can now prove the following result about a generalized $\Lambda_{p}$. 
Theorem 6. Let $\Lambda_{\alpha, \beta}(\rho)=\operatorname{Tr}(\rho) \mathbb{1}+\alpha \rho+\beta \tilde{\rho}_{A}$ be a family of maps. Let $\alpha \geq \max [-1, \beta / 2-1]$ and $\beta \geq \max [-1, \alpha / 2-1]$. Then $\rho \geq 0 \Rightarrow \Lambda_{\alpha, \beta}(\rho)=: \sigma \in \Sigma$, i.e., $\sigma$ is separable.

Proof. The proof is very similar to that of Theorem 2 as above. It is enough to prove it for pure states, $\rho=|\Psi\rangle\langle\Psi|$. Without any loss of generality, take $|\Psi\rangle=\lambda_{0}|0\rangle \otimes|0\rangle+$ $\lambda_{1}|1\rangle \otimes|1\rangle$. It is enough to check then positivity and separability of $\Lambda(|\Psi\rangle\langle\Psi|)$ on a $2 \otimes 2$ space spanned by $|0\rangle,|1\rangle$ in both Alice's and Bob's spaces, where PPT provides then necessary and sufficient condition. After some algebra we obtain the desired condition, represented graphically in Fig. 1, where we mark the range of $\alpha, \beta$ for which $\Lambda_{\alpha, \beta}(\rho)$ is separable for any $\rho$.

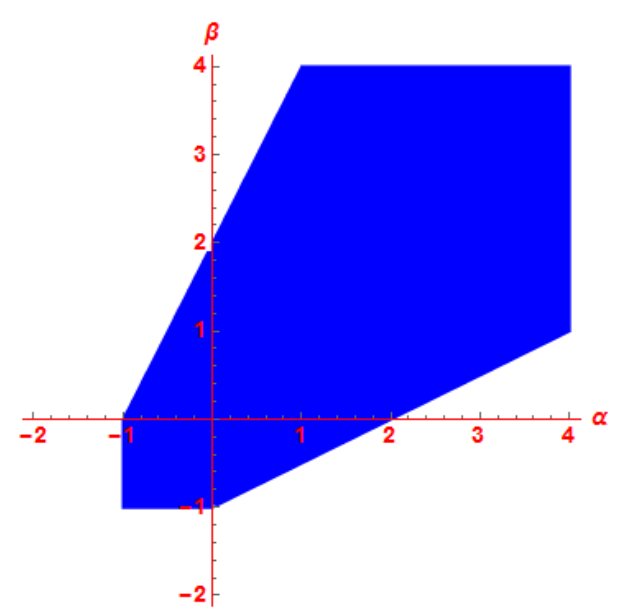

FIG. 1. The range of $\alpha, \beta$ such that for any state $\rho$ acting on $\mathbb{C}^{2} \otimes \mathbb{C}^{N}, \Lambda_{\alpha, \beta}(\rho)$ is separable.

The general Theorem 1 then immediately implies the following separability result.

Theorem 7. (Sufficient separability criterion 2) Let $\rho=$ $\Lambda_{\alpha, \beta}^{-1}(\sigma) \geq 0$, i.e.,

$$
\frac{1}{\alpha^{2}-\beta^{2}}\left[\alpha \sigma-\beta \tilde{\sigma}_{A}-\frac{(\alpha-\beta)}{2 N+\alpha+\beta} \operatorname{Tr}(\sigma) \mathbb{1}\right] \geq 0 .
$$

Then $\sigma$ is separable.

Noticeably, the contributions from $\alpha$ and $\beta$ compensate each other, diminishing in effect off-diagonal parts of $\sigma$ in the Alice space. In fact, for $\alpha=\beta$ these off-diagonal parts strictly vanish and $\sigma$ is a sum of two simple tensor product matrices-in this special case, however, the map ceases to be invertible. The criterion derived above is particularly strong at the boundary of the parameter region represented in Fig. 1, i.e., for $\beta=\alpha / 2-1$ or $\alpha=\beta / 2-1$.

\section{Corrolary 8. (Sufficient separability criterion 3) Let}

$$
\left[\alpha \sigma-(\alpha / 2-1) \tilde{\sigma}_{A}-\frac{(1+\alpha / 2)}{2 N+3 \alpha / 2-1} \operatorname{Tr}(\sigma) \mathbb{1}\right] \geq 0
$$

or

$$
\left[\beta \tilde{\sigma}_{A}-(\beta / 2-1) \sigma-\frac{(1+\beta / 2)}{2 N+3 \beta / 2-1} \operatorname{Tr}(\sigma) \mathbb{1}\right] \geq 0 .
$$

Then $\sigma$ is separable.

The above corollary has a particularly interesting limit, when both $\alpha$ and $\beta$ tend to $\infty$.

\section{Corrolary 9. (Sufficient separability criterion 4) Let}

$$
\sigma-\tilde{\sigma}_{A} / 2>0
$$

or

$$
\tilde{\sigma}_{A}-\sigma / 2>0
$$

Then $\sigma$ is separable.

Note that the latter inequalities are valid only in the asymptotic sense and require strict positivity. One should also note that in the above results we used maps that explicitly involved partially transposed matrices. For this reason it is useful to remind the reader some results of Ref. [30] that hold for $M=2$.

Theorem 10. [30] If $\sigma=\sigma^{T_{A}} \geq 0$ then $\sigma$ is separable.

Corrolary 11. [30] Let $\sigma \geq 0$ be a state. If $\sigma+\sigma^{T_{A}}$ is of full rank and $\left\|\left(\sigma+\sigma^{T_{A}}\right)^{-1}\right\|\left\|\sigma-\sigma^{T_{A}}\right\| \leq 1$, then $\sigma$ is separable. This corollary implies that if $\sigma$ is of full rank and is very close to $\sigma^{T_{A}}$ then $\sigma$ is separable. Here we use the operator norm $\|A\|:=\max _{|| \Psi\rangle \|=1} \| A|\Psi\rangle \|$.

Note that the present results are clearly independent since they involve matrices $\tilde{\sigma}_{A}$ and related ones. Nonetheless, explicit examples showing the independence will be given in Section IV C.

Let us now present the strongest theorem of this section, which involves Breuer-Hall unitary operators on the both sides of Alice and Bob:

Theorem 12. Let $\mathbf{p}=\{\alpha, \beta, \gamma, \delta\}$ and $\Lambda_{\mathbf{p}}(\rho)=\operatorname{Tr}(\rho) \mathbb{1}+\alpha \rho+$ $\beta \tilde{\rho}_{A}+\gamma \tilde{\rho}_{B}+\delta \tilde{\rho}$ be the family of maps; let there exist $0 \leq a, b \leq 1$, $a+b \leq 1$ such that the parameters fulfill the four conditions:

$$
\begin{aligned}
& \alpha \geq \beta / 2-a, \\
& \beta \geq \alpha / 2-a, \\
& \gamma \geq \delta / 2-b, \\
& \delta \geq \gamma / 2-b,
\end{aligned}
$$

and $\alpha \geq-1, \beta \geq-1, \gamma \geq-1$, and $\delta \geq-1$. Then $\rho \geq 0 \Rightarrow$ $\sigma=\Lambda_{\mathbf{p}}(\rho) \in \Sigma$, i.e. $\sigma$ is separable.

Proof. The proof is similar to the proof of Theorem 6, but more complex and technical. Again it is enough to prove for pure states, $\rho=|\Psi\rangle\langle\Psi|$ and, without loosing generality, take $|\Psi\rangle=\lambda_{0}|0\rangle \otimes|0\rangle+\lambda_{1}|1\rangle \otimes|1\rangle$. But, now we have to consider three cases: i) when the Breuer-Hall unitary $V$ acts as $\sigma_{2}$ on the Bob's subspace spanned by $|0\rangle$ and $|1\rangle$; ii) when $V$ transforms $|0\rangle$ and $|1\rangle$ to $|2\rangle$ and $|3\rangle$; iii) when $V$ 
transforms $|0\rangle$ and $|1\rangle$ to orthogonal vectors in a subspace spanned by $|0\rangle$ and $|2\rangle$, and $|1\rangle$ and $|3\rangle$, respectively.

The conditions $\alpha \geq-1, \beta \geq-1, \gamma \geq-1$, and $\delta \geq-1$ follow again from the analysis of the case when $\rho$ is a projector on a product state, say $|0\rangle \otimes|0\rangle$, so that $\tilde{\rho}_{A}$ is a projector on $|1\rangle \otimes|0\rangle, \tilde{\rho}_{B}$ on $|0\rangle \otimes|\tilde{1}\rangle$, and $\tilde{\rho}$ on $|1\rangle \otimes|\tilde{1}\rangle$, where $|\tilde{1}\rangle$ is a vector orthogonal to $|0\rangle$. To derive the conditions in Eq. (10), we split $\mathbb{1}=a \mathbb{1}+b \mathbb{1}+(1-a-b) \mathbb{1}$ and apply the result of Theorem 6 to $a \mathbb{1}+\alpha \rho+\beta \tilde{\rho}_{A}$, and $(1-a) \mathbb{1}+\gamma \tilde{\rho}_{B}+\delta \tilde{\rho}$. Clearly, this estimate of the region of parameters, where $\Lambda_{\mathbf{p}}(\rho)$ is separable, is very conservative, and probably can be improved significantly.

The conditions above are sufficient for separability and correspond to the case iii), which probably is not the most demanding, but we were not able to find weaker sufficient conditions, i.e., the largest allowed regions of the parameters. The case i) leads to the less demanding restrictions that follow from the above conditions, when we set $a+b=1$. Obviously, this case is of interests per $s e$, since it corresponds to exact conditions in the case of two qubits, so that we present it as a separate corollary below. Finally, the case ii) is the least restrictive - indeed it allows to exceed the restriction $a+b \leq 2$ and reach $a=b=1$.
Corrolary 13. Let $\Lambda_{\mathbf{p}}(\rho)=\operatorname{Tr}(\rho) \mathbb{1}+\alpha \rho+\beta \tilde{\rho}_{A}+\gamma \tilde{\rho}_{B}+\delta \tilde{\rho}$ be the family of maps acting on $\mathcal{H}=\mathcal{H}_{A} \otimes \mathcal{H}_{B}=\mathbb{C}^{2} \otimes \mathbb{C}^{2}$, with $V=\sigma_{2}$ (the second Pauli matrix) acting in the Bob's space. Let $s=\alpha+\gamma, \tilde{s}=\beta+\delta$, and let the parameters fulfill the conditions:

$$
\begin{aligned}
& s \geq \max [-1, \tilde{s} / 2-1], \\
& \tilde{s} \geq \max [-1, s / 2-1],
\end{aligned}
$$

and $\alpha \geq-1, \beta \geq-1, \gamma \geq-1$, and $\delta \geq-1$. Then if $\rho \geq 0$, $\sigma=\Lambda_{\mathbf{p}}(\rho) \in \Sigma$, i.e. $\sigma$ is separable.

Proof. The proof is straightforward, since, PPT provides the if and only if criterion for separability in the case 2 by 2. We take $\rho=|\Psi\rangle\langle\Psi|$ with $|\Psi\rangle=\lambda_{0}|0\rangle \otimes|0\rangle+\lambda_{1}|1\rangle \otimes|1\rangle$, and simply write down the matrices $\Lambda_{\mathbf{p}}(\rho)$ and $\Lambda_{\mathbf{p}}(\rho)^{T_{A}}$ and check positive definiteness. We observe that it is enough to consider the "extreme" cases, when either $\lambda_{0}=0$ or $\lambda_{1}=0$, or when $|\Psi\rangle$ is maximally entangled, i.e. $\lambda_{0}=$ $\lambda_{1}=1 / \sqrt{2}$. The latter case gives us conditions for $s$ and $\tilde{s}$, whereas the former the simple lower bounds by -1 on all parameters. To be more explicit the conditions $\alpha \geq-1$, $\beta \geq-1, \gamma \geq-1$, and $\delta \geq-1$ follow from the analysis of the case when $\rho$ is a projector on a product state, say $|0\rangle \otimes|0\rangle$, so that $\tilde{\rho}_{A}$ is a projector on $|1\rangle \otimes|0\rangle, \tilde{\rho}_{B}$ on $|0\rangle \otimes|1\rangle$, and $\tilde{\rho}$ on $|1\rangle \otimes|1\rangle$. Let us explicitly write $\Lambda_{\mathbf{p}}(\rho)=\operatorname{Tr}(\rho) \mathbb{1}+\alpha \rho+\beta \tilde{\rho}_{A}+$ $\gamma \tilde{\rho}_{B}+\delta \tilde{\rho}$, for $\rho=|\Psi\rangle\langle\Psi|$ with $|\Psi\rangle=\lambda_{0}|0\rangle \otimes|0\rangle+\lambda_{1}|1\rangle \otimes|1\rangle$ :

$$
\Lambda_{\mathbf{p}}(\rho)=\left(\begin{array}{cccc}
1+\alpha \lambda_{0}^{2}+\delta \lambda_{1}^{2} & 0 & 0 & (\alpha-\beta+\delta-\gamma) \lambda_{0} \lambda_{1} \\
0 & 1+\gamma \lambda_{0}^{2}+\beta \lambda_{1}^{2} & 0 & 0 \\
0 & 0 & 1+\beta \lambda_{0}^{2}+\gamma \lambda_{1}^{2} & 0 \\
(\alpha-\beta+\delta-\gamma) \lambda_{0} \lambda_{1} & 0 & 0 & 1+\delta \lambda_{0}^{2}+\alpha \lambda_{1}^{2}
\end{array}\right)
$$

It is easy to observe that the positivity of $\Lambda_{\mathbf{p}}(\rho)$ and its partial transpose (i.e. its separability), gives the strongest restrictions on the parameters when $|\Psi\rangle$ is maximally entangled, i.e. $\lambda_{0}=\lambda_{1}=1 / \sqrt{2}$. Direct inspection then leads to the conditions in Eq. (11).

Note that the proof of the Theorem 6 is just the same, when we set $\gamma=\delta=0$, while the proof of the Theorem 12 , as discussed above, is more involved.

\section{B. Ando-like maps}

So far we were mainly generalizing the Breuer-Hall type maps in the context of our general approach. Let us now present a similar separability criterion based on another family of positive maps, the so called Ando's maps [31-33]. For $k=1,2, \ldots, d-1$, the $\operatorname{map} \Lambda_{k ; \alpha}: \mathcal{B}\left(\mathbb{C}^{d}\right) \rightarrow$
$\mathcal{B}\left(\mathbb{C}^{d}\right)$ is defined by

$$
\Lambda_{k ; \alpha}(\rho):=(d-k) \epsilon(\rho)+\sum_{l=1}^{k} \epsilon\left(S^{l} \rho S^{l \dagger}\right)+\alpha \rho,
$$

where $S|i\rangle=|i+1\rangle$ is a unitary shift modulo $d$, and $\epsilon(X):=\sum_{i=0}^{d-1}|i\rangle\langle i|X| i\rangle\langle i|$. It is known that $\Lambda_{k ; \alpha}$ is positive for $\alpha \geq-1$ [31-33]. Also note that $\Lambda_{d-1 ; \alpha}=\mathbb{1} \operatorname{Tr}(\rho)+\alpha \rho$ is the generalized reduction map already considered in Theorem 2.

The Ando-type maps defined above do not consider the internal tensor-product structure of the space $\mathcal{B}\left(\mathbb{C}^{d}\right)$. To make it transparent, we define for $\rho \in \mathcal{B}\left(\mathbb{C}^{2} \otimes \mathbb{C}^{N}\right)$ and $-1 \leq k \leq N-1$,

$$
\begin{aligned}
\Lambda_{k, \alpha}^{2 \times N}(\rho) & :=(N-k-1) \epsilon(\rho)+\sum_{m=0}^{N-1} \epsilon\left(S_{B}^{m} \rho S_{B}^{m^{\dagger}}\right) \\
& +\sum_{m=0}^{k} \epsilon\left(S_{A} S_{B}^{m} \rho S_{A}^{\dagger} S_{B}^{m^{\dagger}}\right)+\alpha \rho,
\end{aligned}
$$


where

$$
\epsilon(X):=\sum_{i=0, j=0}^{1, N-1}|i, j\rangle\langle i, j|X| i, j\rangle\langle i, j|,
$$

and $S_{A}, S_{B}$ are the unitary shift operator on the Alice and Bob spaces, respectively. The case $k=-1$ is particularly simple, since the unitary shift is applied on Bob's side only. For simplicity, we denote it differently, $\Lambda_{-1}^{2 \times N}(.) \equiv$ $\Lambda^{2 \times N}($.), and discuss below its separability properties. Its generalization for $M \geq 3$ is presented in Section VB.
Theorem 14. For $\rho \in \mathcal{B}\left(\mathbb{C}^{2} \otimes \mathbb{C}^{N}\right)$,

$$
\sigma:=\Lambda^{2 \times N}(\rho)=N \epsilon(\rho)+\sum_{m=0}^{N-1} \epsilon\left(S_{B}^{m} \rho S_{B}^{m \dagger}\right)+\alpha \rho
$$

is separable if $-2 N /(3 N-1) \leq \alpha \leq 1$.

Proof. As usual, we can restrict ourselves to consider $\rho=$ $|\psi\rangle\langle\psi|$, and without loosing generality assume that $|\psi\rangle=$ $|0\rangle|e\rangle+|1\rangle|f\rangle$, with $|e\rangle=\sum_{j=0}^{N-1} \lambda_{0 j}|j\rangle,|f\rangle=\sum_{j=0}^{N-1} \lambda_{1 j}|j\rangle$, and $\sum_{j=0}^{N-1}\left(\left|\lambda_{0 j}\right|^{2}+\left|\lambda_{1 j}\right|^{2}\right)=1$. One gets (we present the results for $N=3$, but generalization for $N>3$ is straightforward)

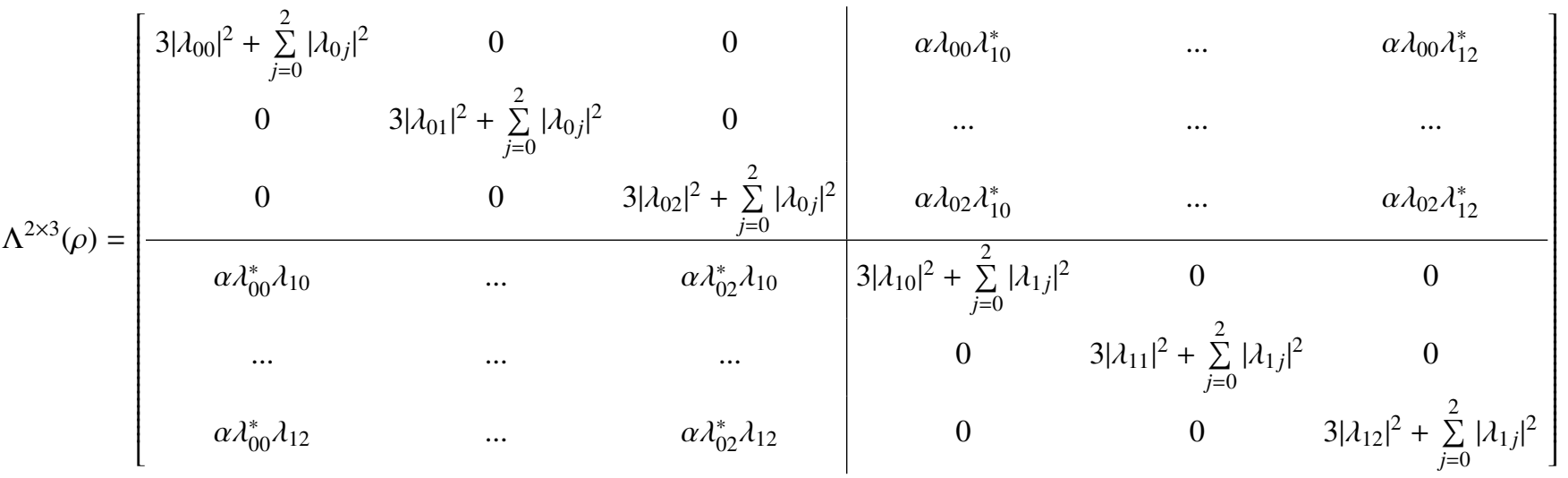

$$
\begin{aligned}
& +\alpha|0\rangle\langle 0|\otimes| e\rangle\langle e|+\alpha| 1\rangle\langle 1|\otimes| f\rangle\langle f| .
\end{aligned}
$$

The result is separable iff it is non-negative and PPT, but in the proof we will not use the PPT for the sake of generalization. Instead, we subtract from $\Lambda^{2 \times 3}(\rho)$ a set of explicitly separable (unnormalized) states $\sigma_{i, j}$ (with $i<j=0,1,2$ ), which are supported in $2 \otimes 2$ (thus nonnegativity and PPT implying separability) and are of the form

$$
\sigma_{i, j}=\left[\begin{array}{cc|cc}
|\alpha|\left(\left|\lambda_{0 i}\right|^{2}+\left|\lambda_{0 j}\right|^{2}\right) & 0 & 0 & \alpha \lambda_{0 i} \lambda_{1 j}^{*} \\
0 & |\alpha|\left(\left|\lambda_{0 i}\right|^{2}+\left|\lambda_{0 j}\right|^{2}\right) & \alpha \lambda_{0 j} \lambda_{1 i}^{*} & 0 \\
\hline 0 & \alpha \lambda_{0 j}^{*} \lambda_{1 i} & |\alpha|\left(\left|\lambda_{1 i}\right|^{2}+\left|\lambda_{1 j}\right|^{2}\right) & 0 \\
\alpha \lambda_{0 i}^{*} \lambda_{1 j} & 0 & 0 & |\alpha|\left(\left|\lambda_{1 i}\right|^{2}+\left|\lambda_{1 j}\right|^{2}\right)
\end{array}\right] .
$$

The remainder $R(\rho):=\Lambda^{2 \times 3}(\rho)-\sum_{i<j=0}^{2} \sigma_{i, j}$ is given by

$$
R(\rho)=\left[\begin{array}{c|c}
D_{0} & X \\
\hline X^{\dagger} & D_{1}
\end{array}\right]+\alpha|0\rangle\langle 0|\otimes| e\rangle\langle e|+\alpha| 1\rangle\langle 1|\otimes| f\rangle\langle f|,
$$

$$
\text { where } \begin{aligned}
D_{k} & =\operatorname{diag}\left\{(3-|\alpha|)\left|\lambda_{k i}\right|^{2}+(1-|\alpha|) N_{k}\right\}_{i=0}^{2}, k=0,1, \\
X & =\operatorname{diag}\left\{\alpha \lambda_{00} \lambda_{10}^{*}, \alpha \lambda_{01} \lambda_{11}^{*}, \alpha \lambda_{02} \lambda_{12}^{*}\right\} \\
N_{0} & =1-N_{1}=\sum_{j=0}^{2}\left|\lambda_{0 j}\right|^{2} .
\end{aligned}
$$

For $0 \leq \alpha \leq 1, R(\rho)$ is explicitly non-negative and separable. For $\alpha<0$, we again subtract separable states of the form $|\alpha|\left|e_{i}\right\rangle\left\langle e_{i}|\otimes| i\right\rangle\langle i|(i=0,1,2)$, with $\left|e_{i}\right\rangle=\lambda_{0 i}|0\rangle-\lambda_{1 i}|1\rangle$, 
and end up with

$$
R^{\prime}(\rho)=\left[\begin{array}{c|c}
D_{0}^{\prime} & \mathbf{0} \\
\hline \mathbf{0} & D_{1}^{\prime}
\end{array}\right]+\alpha|0\rangle\langle 0|\otimes| e\rangle\langle e|+\alpha| 1\rangle\langle 1|\otimes| f\rangle\langle f|,
$$

where $D_{k}^{\prime}=\operatorname{diag}\left\{(3-2|\alpha|)\left|\lambda_{k i}\right|^{2}+(1-|\alpha|) N_{k}\right\}_{i=0}^{2}, k=0,1$.

Noticing that the RHS of Eq. (15) could be written as $|0\rangle\left\langle 0\left|\otimes\left(D_{0}^{\prime}+\alpha|e\rangle\langle e|\right)+\right| 1\right\rangle\langle 1| \otimes\left(D_{1}^{\prime}+\alpha|f\rangle\langle f|\right), R^{\prime}(\rho)$ is explicitly separable if $D_{0}^{\prime}+\alpha|e\rangle\langle e| \geq 0$ and $D_{1}^{\prime}+\alpha|f\rangle\langle f| \geq 0$.

After straightforward calculations using the results of Ref. [34], we get that this requires that for all choices of $\lambda$ 's we must have

$$
\sum_{j=0}^{N-1} \frac{|\alpha|\left|\lambda_{0 j}\right|^{2}}{(3-2|\alpha|)\left|\lambda_{0 j}\right|^{2}+(1-|\alpha|) N_{0}} \leq 1 .
$$

This leads to $-3 / 4 \leq \alpha$, or generally $-2 N /(3 N-1) \leq \alpha$. Numerical analysis and examples suggest that the regions of $\alpha$ assuring separability can be extended to $-1 \leq$ $\alpha$, but we were not able to prove it.

Noticing that the map $\Lambda^{2 \times N}$ allows an inverse, the above theorem implies the following sufficient separability criterion.

Corrolary 15. (Sufficient separability criterion 5) Let $-2 N /(3 N-1) \leq \alpha \leq 1$ and

$\frac{1}{\alpha}\left(\sigma-\frac{N}{N+\alpha} \epsilon(\sigma)-\frac{\alpha}{(N+\alpha)(2 N+\alpha)} \sum_{m=0}^{N-1} \epsilon\left(S_{B}^{m} \sigma S_{B}^{m \dagger}\right)\right) \geq 0$.

Then $\sigma$ is separable.
The methods used to prove Theorem 14 can readily be applied to prove the cases of other $k^{\prime}$ s. The next result is such a generalization.

Theorem 16. For $\rho \in \mathcal{B}\left(\mathbb{C}^{2} \otimes \mathbb{C}^{N}\right), \Lambda_{k}^{2 \times N}(\rho)$ is separable if

- $k=0$ and $-(2 N-1) /(3 N-2) \leq \alpha \leq 1$

- $k=1$ and $-(2 N-2) /(3 N-2) \leq \alpha \leq 1$

- for arbitrary $k$ and $-(2 N-k-1) /(3 N-2) \leq \alpha \leq 1$

- $k=N-1$ and $-1 \leq \alpha \leq 2$ (reduction map)

Proof. The case $k=N-1$ follows from the results above. We provide first the proof for $k=0$ and $N=3$-generalization to $k=0$ and $N>3$ is straightforward. We follow exactly the steps from the proof of Theorem 14 and first subtract the matrices $\sigma_{i, j}$ defined in Eq. (13) from $\Lambda_{0}^{2 \times 3}(\rho)$ to obtain

$$
R^{\prime \prime}(\rho)=\left[\begin{array}{c|c}
D_{0}^{\prime \prime} & X \\
\hline X^{\dagger} & D_{1}^{\prime \prime}
\end{array}\right]+\alpha|0\rangle\langle 0|\otimes| e\rangle\langle e|+\alpha| 1\rangle\langle 1|\otimes| f\rangle\langle f|,
$$

where

$$
\begin{aligned}
D_{k}^{\prime \prime}=\operatorname{diag}\{ & (2-|\alpha|)\left|\lambda_{00}\right|^{2}+(1-|\alpha|) N_{0}+\left|\lambda_{10}\right|^{2}, \\
& (2-|\alpha|)\left|\lambda_{01}\right|^{2}+(1-|\alpha|) N_{0}+\left|\lambda_{11}\right|^{2}, \\
& \left.(2-|\alpha|)\left|\lambda_{02}\right|^{2}+(1-|\alpha|) N_{0}+\left|\lambda_{12}\right|^{2}\right\}
\end{aligned}
$$

and $X$ is defined as in the proof of Theorem 14. Now, for $\alpha \geq 0$ it is not difficult to see that the first matrix in (16) is separable. Thus, $R^{\prime \prime}(\rho)$ is separable and so is $\Lambda_{0}^{2 \times 3}(\rho)$.

For $\alpha<0$, we have to modify the second step a little bit, taking $\left|e_{i}\right\rangle=\lambda_{0 i}|1\rangle-\lambda_{1 i}|0\rangle$. In this way we end up with the condition that $\Lambda_{0}^{2 \times 3}(\rho)$ is explicitly separable if

$$
\left[\begin{array}{ccc}
(2-|\alpha|)\left|\lambda_{00}\right|^{2}+(1-|\alpha|)\left(N_{0}+\left|\lambda_{10}\right|^{2}\right) & 0 & 0 \\
0 & (2-|\alpha|)\left|\lambda_{01}\right|^{2}+(1-|\alpha|)\left(N_{0}+\left|\lambda_{11}\right|^{2}\right) & 0 \\
0 & 0 & (2-|\alpha|)\left|\lambda_{02}\right|^{2}+(1-|\alpha|)\left(N_{0}+\left|\lambda_{12}\right|^{2}\right)
\end{array}\right]+\alpha|e\rangle\langle e| \geq 0
$$

and the similar condition holds for the $|f\rangle\langle f|$ part. The calculations using the results of Ref. [34] are similar; we get that $|\alpha|$ must fulfill for all choices of $\lambda^{\prime} \mathrm{s}$

$$
\sum_{j=0}^{N-1} \frac{\left|\alpha \| \lambda_{0 j}\right|^{2}}{(2-|\alpha|)\left|\lambda_{0 j}\right|^{2}+(1-|\alpha|)\left(N_{0}+\left|\lambda_{1 j}\right|^{2}\right)} \leq 1
$$

and the analogous conditions for the $|f\rangle\langle f|$ part. It is easy to see that the bound on $|\alpha|$ is $-5 / 7 \leq \alpha$, or in general $-(2 N-1) /(3 N-2) \leq \alpha$. This kind of proof works indeed for arbitrary $k$ and leads in general to the condition $-(2 N-1-k) /(3 N-2) \leq \alpha$. Clearly, as $k$ approaches $N-1$ we expect that a better proof and better estimates should be possible, but we have not found them.

\section{Examples}

In this subsection we will present examples of separable states detected by our criteria. For the sake of comparison, we will consider other separability criteria from the literature. As such, the basic standard is to compare with the results from Ref. [24]. The strongest separability criterion derivable from Theorem 1 of Ref. [24] is the Corollary 2 (scaling criterion) therein, which for a normalized $M \otimes N$ state $\rho$ becomes the purity condition,

$$
\operatorname{Tr}\left(\rho^{2}\right) \leq 1 /(M N-1) \Rightarrow \rho \in \Sigma \text {. }
$$

States satisfying Eq. (18) are usually termed as members of the separable ball around identity. Since our criteria 
Eqs. (5)-(9) are mainly for $2 \otimes N$ states, we use the relation $\tilde{\sigma}_{A}:=\sigma_{2} \sigma^{T_{A}} \sigma_{2}=\mathbb{1} \otimes \sigma_{B}-\sigma$ to simplify the equations a little bit. Then Eqs. (6)-(7) become

$$
\begin{aligned}
& \left(3 \frac{\alpha}{2}-1\right) \sigma \geq\left(\frac{\alpha}{2}-1\right) \mathbb{1} \otimes \sigma_{B}+\frac{1+\alpha / 2}{2 N+3 \alpha / 2-1} \mathbb{1} \\
& \left(3 \frac{\beta}{2}-1\right) \sigma \leq \beta \mathbb{1} \otimes \sigma_{B}-\frac{1+\beta / 2}{2 N+3 \beta / 2-1} \mathbb{1}
\end{aligned}
$$

where $0 \leq \alpha, \beta<\infty$. If we set $\alpha=2$ (so that $\beta=0$ ), Eq. (19a) becomes the separability criterion mentioned in Corrolary 4.

The first bound entangled state in the literature (and also in the least possible dimension, $2 \otimes 4$ ) is given by [35]

$$
\begin{gathered}
\rho_{a}=\frac{7 a}{7 a+1} \rho_{\mathrm{ent}}+\frac{1}{7 a+1}|\phi\rangle\langle\phi|, \quad a \in[0,1], \\
\phi\rangle=|1\rangle \otimes\left(\sqrt{\frac{1+a}{2}}|0\rangle+\sqrt{\frac{1-a}{2}}|2\rangle\right), \\
\rho_{\mathrm{ent}}=\frac{2}{7} \sum_{i=1}^{3}\left|\psi_{i}\right\rangle\left\langle\psi_{i}\left|+\frac{1}{7}\right| 03\right\rangle\langle 03|, \\
\left|\psi_{i}\right\rangle=\frac{1}{\sqrt{2}}(|0\rangle|i-1\rangle+|1\rangle|i\rangle), \quad i=1,2,3 .
\end{gathered}
$$

The state $\rho_{a}$ remains PPT throughout $a \in[0,1]$ and for $0<a<1$, it is bound entangled.

Let us now consider the following class of states,

$$
\rho_{a, p}=p \rho_{a}+\frac{1-p}{8} \mathbb{1}_{8}
$$

One verifies (e.g., with a computer program) that for all $a, p$ in the range

$$
0<a<\frac{1}{224}(\sqrt{3745}-49), \frac{1}{7} \sqrt{\frac{343 a^{2}+98 a+7}{47 a^{2}-6 a+7}}<p \leq \frac{1}{5},
$$

$\rho_{a, p}$ violates Eq. (18) but satisfies Eq. (19a) with $\alpha=2$. Hence, $\rho_{a, p}$ lies outside the separable ball, but still detected to be separable by the criterion mentioned in Corrolary 4. Similar examples could be constructed with other values of $\alpha$. However, note that no state $\rho_{a, p}$ lying outside the separable ball could be detected by Eqs. (8)(9).

\section{Non-invertible maps}

In this subsection we will consider (sufficient) separability criterion arising from some non-invertible maps. Due to non-invertibility of the maps involved, the derived criteria would not follow from the general theorem of this paper. It is quite challenging to generalize them to the invertible case.
Our first example is about the map $\Phi_{\alpha}: \mathcal{B}\left(\mathbb{C}^{2} \otimes \mathbb{C}^{N}\right) \rightarrow$ $\mathcal{B}\left(\mathbb{C}^{2} \otimes \mathbb{C}^{N}\right)$ defined as follows: any Hermitian $X \in$ $\mathcal{B}\left(\mathbb{C}^{2} \otimes \mathbb{C}^{N}\right)$ may be written as

$$
X=\left(\begin{array}{ll}
X_{11} & X_{12} \\
X_{12}^{\dagger} & X_{22}
\end{array}\right),
$$

where $X_{i j} \in \mathcal{B}\left(\mathbb{C}^{N}\right)$. Let

$$
\Phi_{\alpha}(X):=\left(\begin{array}{cc}
\mathbb{I}_{N} \operatorname{Tr} X_{11} & \alpha B \\
\alpha B^{\dagger} & \mathbb{I}_{N} \operatorname{Tr} X_{22}
\end{array}\right)
$$

with $B=X_{12}+R_{N}\left(X_{12}^{\dagger}\right)$ and $R_{N}: \mathcal{B}\left(\mathbb{C}^{N}\right) \rightarrow \mathcal{B}\left(\mathbb{C}^{N}\right)$ being the reduction map. It was shown in Ref. [36] that $\Phi_{\alpha=1}$ defines an optimal positive map. Clearly, $\Phi_{\alpha}$ is positive for all $|\alpha| \leq 1$ (it is optimal for $|\alpha|=1$ ).

Theorem 17. $\Phi_{\alpha}(|\psi\rangle\langle\psi|)$ is separable if $|\alpha| \leq 1$.

Proof. The proof follows directly from Proposition 1 of Ref. [24], since $\Phi_{\alpha}(|\psi\rangle\langle\psi|)$ is locally equivalent to

$$
\left(\begin{array}{ll}
\mathbb{I}_{N} & A \\
A^{\dagger} & \mathbb{I}_{N}
\end{array}\right)
$$

with $A A^{\dagger} \leq \mathbb{I}_{N}$.

We now consider a slightly generalized map $\Psi_{\alpha}: \mathcal{B}\left(\mathbb{C}^{4} \otimes \mathbb{C}^{N}\right) \rightarrow \mathcal{B}\left(\mathbb{C}^{4} \otimes \mathbb{C}^{N}\right)$ defined as follows: any Hermitian $X \in \mathcal{B}\left(\mathbb{C}^{4} \otimes \mathbb{C}^{N}\right)$ may be written as

$$
X=\left(\begin{array}{ll}
X_{11} & X_{12} \\
X_{12}^{\dagger} & X_{22}
\end{array}\right)
$$

where $X_{i j} \in \mathcal{B}\left(\mathbb{C}^{2} \otimes \mathbb{C}^{N}\right)$. Let

$$
\Psi_{\alpha}(X):=\left(\begin{array}{cc}
\mathbb{I}_{2 N} \operatorname{Tr} X_{11} & \alpha C \\
\alpha C^{\dagger} & \mathbb{I}_{2 N} \operatorname{Tr} X_{22}
\end{array}\right)
$$

with $C=X_{12}+U \overline{X_{12}} U^{\dagger}, U$ being an arbitrary antisymmetric $2 N \times 2 N$ unitary matrix. It is known [37] that $\Psi_{\alpha=1}$ defines an optimal positive map. It is also clear that $\Psi_{\alpha}$ is positive for all $|\alpha| \leq 1$ (it is optimal for $|\alpha|=1$ ).

Theorem 18. $\Psi_{\alpha}(|\psi\rangle\langle\psi|)$ is separable if $|\alpha| \leq 1$.

The proof is essentially the same as that of Theorem 17.

\section{MAIN RESULTS FOR $\mathbb{C}^{M} \otimes \mathbb{C}^{N}$}

\section{A. Reduction- and Breuer-Hall-like maps}

There is a general and simple generalization of the Theorem 1, summarized here as the Corollary. 
Corrolary 19. Let $\mathbf{p}=\{\alpha, \beta, \gamma, \delta\}$ and $\Lambda_{\mathbf{p}}(\rho)=\operatorname{Tr}(\rho) \mathbb{1}+$ $\alpha \rho+\beta \tilde{\rho}_{A}+\gamma \tilde{\rho}_{B}+\delta \tilde{\rho}$ be a family of maps with the parameters fulfilling the conditions such that $\rho \geq 0 \Rightarrow \Lambda_{\mathbf{p}}(\rho) \in \Sigma$. Then, if $w=P+Q^{T_{A}}$ is a decomposable pre-witness with $P, Q \geq 0$, $\Lambda_{\mathbf{p}}(w)=: \sigma \in \Sigma$, i.e. $\sigma$ is also separable.

Proof. The proof follows easily from the fact that $\Lambda_{\mathbf{p}}\left(w^{T_{A}}\right)=\Lambda_{\mathbf{p}}(w)^{T_{A}}$. We present this corollary in this section since it holds in $\mathbb{C}^{M} \otimes \mathbb{C}^{N}$ equally well as in $\mathbb{C}^{2} \otimes \mathbb{C}^{N}$, provided appropriate generalizations of BreuerHall unitary operators are used.

Let us now present our own proof of the Theorem 2, i.e., separability of $\Lambda_{\alpha}(\rho)=\operatorname{Tr}(\rho) \mathbb{1}+\alpha \rho$ for arbitrary $M \otimes N$ $\rho$.

Proof. We discuss in detail the cases $M=3,4-$ the generalizations to arbitrary $M \leq N$ is the straightforward. As before, it is enough to prove for pure states, $\rho=|\Psi\rangle\langle\Psi|$. For $M=3,|\Psi\rangle$ has maximally Schmidt rank 3 , and, without loosing generality, we can assume that $|\Psi\rangle=\lambda_{0}|0\rangle \otimes|0\rangle+\lambda_{1}|1\rangle \otimes|1\rangle+\lambda_{2}|2\rangle \otimes|2\rangle$. It is enough to check then positivity and separability of $\Lambda(|\Psi\rangle\langle\Psi|)$ on a $3 \otimes 3$ space spanned by $|0\rangle,|1\rangle$ and $|2\rangle$ in both Alice's and Bob's spaces. This is achieved by observing first that if $|\Psi\rangle$ is a normalized state, than obviously positivity of $\Lambda_{\alpha}(|\Psi\rangle\langle\Psi|)$ requires $\alpha \geq-1$. Direct inspection shows that for $\alpha=-1, \Lambda_{\alpha}(|\Psi\rangle\langle\Psi|)$ is a sum of three positive matrices that effectively act on two-qubit Hilbert spaces and are given by

$$
\sigma_{i j}=\left|\varphi_{i j}\right\rangle\left\langle\varphi_{i j}|+| i j\right\rangle\langle i j|+| j i\rangle\langle j i|,
$$

where $\left|\varphi_{i j}\right\rangle=\lambda_{j}|i\rangle-\lambda_{i}|j\rangle$ with $i<j=0,1,2$. These matrices are PPT and thus separable.

For $\alpha \geq 0$, we decompose $\Lambda_{\alpha}(|\Psi\rangle\langle\Psi|)=\sigma^{T_{A}}+D$, where $\sigma^{T_{A}}$ is separable by construction, and $D$ is positive diagonal in the computational product basis, ergo separable by direct inspection. We consider a family of product vectors $|p(\phi, \psi)\rangle=A\left(1, a e^{i \phi}, b e^{i \psi}\right)^{\otimes 2}$ and the separable state

$$
\sigma=\int d \phi / 2 \pi \int d \psi / 2 \pi|p(\phi, \psi)\rangle\langle p(\phi, \psi)| .
$$

The parameters can be chosen indeed in such a way that $\Lambda_{\alpha}(|\Psi\rangle\langle\Psi|)-\sigma^{T_{A}}$ is diagonal. To this aim we choose $A^{2}=$ $\alpha \lambda_{0}^{2}, a^{2}=\lambda_{1} / \lambda_{0}, b^{2}=\lambda_{2} / \lambda_{0}$. Checking the explicit conditions that $d$ is positive implies that $1-A^{2} a^{2}=1-\alpha \lambda_{0} \lambda_{1} \geq 0$, i.e. $\alpha \leq 2$ since $\lambda_{0}$ and $\lambda_{1}$ are the highest and the second highest Schmidt coefficients, respectively. The case $-1<\alpha<0$ follows from convexity.

The proof for the case $M=4$ is analogous. We have now four Schmidt coefficients, $\lambda_{k}, k=0, \ldots, 3$, and take $|p(\phi, \psi)\rangle=A\left(1, a e^{i \phi}, b e^{i \psi}, c e^{i \theta}\right)^{\otimes 2}$, set $A^{2}=\alpha \lambda_{0}^{2}, a^{2}=\lambda_{1} / \lambda_{0}$, $b^{2}=\lambda_{2} / \lambda_{0}$, and $c^{2}=\lambda_{3} / \lambda_{0}$. Generalization for $M>4$ follows the same pattern.

The above theorem can be generalized to the sufficient conditions for states with Schmidt number $n \leq M$, $\sigma_{n} \in \Sigma_{n}$. Similar results were obtained earlier by L. Clarisse [38], but our proofs are different, so we present them here.
Theorem 20. (see also [38]) Let $\Lambda_{\alpha}(\rho)=\operatorname{Tr}(\rho) \mathbb{1}+\alpha \rho$ be the family of maps, and $-1 \leq \alpha \leq n+1$. Then $\rho \geq 0 \Rightarrow$ $\Lambda_{\alpha}(\rho)=\sigma_{n} \in \Sigma_{n}$, i.e. $\sigma_{n}$ has the Schmidt number $\leq n$.

Proof. We discuss in detail the case $M=3$ and $n=2-$ the generalization to arbitrary $M \leq N$ is the straightforward. As before it is enough to prove for pure states, $\rho=|\Psi\rangle\langle\Psi|$. For $M=3,|\Psi\rangle$ has maximally Schmidt rank 3 , and, without loosing generality, we can assume that $|\Psi\rangle=\lambda_{0}|0\rangle \otimes|0\rangle+\lambda_{1}|1\rangle \otimes|1\rangle+\lambda_{2}|2\rangle \otimes|2\rangle$. It is enough to check then positivity and Schmidt number of $\Lambda(|\Psi\rangle\langle\Psi|)$ on a $3 \otimes 3$ space spanned by $|0\rangle,|1\rangle$ and $|2\rangle$ in both Alice's and Bob's spaces. This is achieved by observing first that if $|\Psi\rangle$ is a normalized state, than obviously positivity of $\Lambda_{\alpha}(|\Psi\rangle\langle\Psi|)$ requires $\alpha \geq-1$. Moreover, we know that for $\alpha=-1, \Lambda_{\alpha}(|\Psi\rangle\langle\Psi|)$ is separable, ergo it obviously has the Schmidt number $1 \leq n=2$. For $\alpha \geq 0$, we decompose directly $\Lambda_{\alpha}(|\Psi\rangle\langle\Psi|)=\sigma^{T_{A}}+D_{2}^{T_{A}}$, where $\sigma$ is separable by construction, and $D_{2}$ has a form that explicitly implies that its Schmidt number is smaller equal 2. As before, we consider a family of product vectors $|p(\phi, \psi)\rangle=A\left(1, a e^{i \phi}, b e^{i \psi}\right)^{\otimes 2}$ and the separable state

$$
\sigma=\int d \phi / 2 \pi \int d \psi / 2 \pi|p(\phi, \psi)\rangle\langle p(\phi, \psi)| .
$$

The parameters can be chosen indeed in such a way that $\Lambda_{\alpha}(|\Psi\rangle\langle\Psi|)-\sigma_{A}^{T}$ has only two off-diagonal elements, corresponding to the subspace spanned by $|0\rangle$ and $|1\rangle$. To this aim we choose $A^{2}=\epsilon \alpha \lambda_{0}^{2}, a^{2}=\lambda_{1} / \lambda_{0}, b^{2}=\lambda_{2} / \lambda_{0} \epsilon$, with some $\epsilon \geq 0$. Checking the explicit conditions that $d$ is positive implies, among others, that $1-A^{2} b^{2}=$ $1-\alpha \lambda_{0} \lambda_{2} \geq 0$, i.e. $\alpha \leq 3$, since $\lambda_{0}$ and $\lambda_{2}$ are the highest and the third highest Schmidt coefficients, respectively. Other conditions, such as $1-A^{2} a^{2}=1-\epsilon \alpha \lambda_{0} \lambda_{1} \geq 0$, or $1+\alpha \lambda_{2}^{2}-A^{2} b^{4}=1+\alpha \lambda_{2}^{2}(1-1 / \epsilon) \geq 0$, can be fulfilled with $\epsilon=2 / 3$, for instance.

Generalization for $M \geq 4$ and $n=2$ is straightforward: we set $A^{2}=\epsilon \alpha \lambda_{0}^{2}, a^{2}=\lambda_{1} / \lambda_{0}, b^{2}=\lambda_{2} / \lambda_{0} \epsilon, c^{2}=\lambda_{3} / \lambda_{0} \epsilon, \ldots$ with some $\epsilon=2 / 3$. For $M \geq 4$ and $n=3$ we set $A^{2}=\epsilon \alpha \lambda_{0}^{2}$, $a^{2}=\lambda_{1} / \lambda_{0}, b^{2}=\lambda_{2} / \lambda_{0}, c^{2}=\lambda_{3} / \lambda_{0} \epsilon$, etc. with $\epsilon=1 / 2$.

Much stronger result than that of Theorem 20 was proven in Ref. [38] for $M=N=d$.

Theorem 21. Let $\Lambda_{\alpha}(\rho)=\operatorname{Tr}(\rho) \mathbb{1}+\alpha \rho$ be a family of maps with $-1 \leq \alpha \leq 2(d n-1) /(d-n)$. Then, if $\rho \geq 0 \Rightarrow \sigma=$ $\Lambda_{\alpha}(\rho) \in \Sigma_{n}$, i.e. $\sigma$ has the Schmidt number $\leq n$.

Proof. Let $|\psi\rangle$ be a two-qudit state with Schmidt coefficients $\lambda_{0} \geq \lambda_{1} \geq \ldots$. It was shown in Ref. [38] that the (unnormalized) mixture of $|\psi\rangle$ and the maximally mixed state, $\mathbb{1}+\alpha|\psi\rangle\langle\psi|$, has Schmidt number $n$ if

$$
\alpha \leq \frac{d n-1}{(d-n) \lambda_{0} \lambda_{1}} .
$$

This implies that $\mathbb{1}+\alpha|\psi\rangle\langle\psi|$ has Schmidt number at most $n$ for any $|\psi\rangle$ if

$$
\alpha \leq \frac{2(d n-1)}{d-n} .
$$


and

The value $2(d n-1) /(d-n)$ is larger than $n+1$ and it recovers the bound $\alpha \leq 2$ for separability. Still, the proof of Theorem 20 is relatively simple and we expect that it could be generalized to other situations, such as the multipartite case etc. That is why we decided to present it here.

Let us finally notice that Theorem 21 implies the following sufficient criterion for being an element of $\Sigma_{n}$, which generalizes Theorem 3 .

Corrolary 22. If for some $\alpha \in[-1,2(d n-1) /(d-n)]$, $\Lambda_{\alpha}^{-1}(\sigma):=[\sigma-\operatorname{Tr}(\sigma) \mathbb{1} /(2 N+\alpha)] / \alpha \geq 0$, then $\sigma \in \Sigma_{n}$, i.e., the Schmidt number of $\rho$ is not larger than $n$.

\section{B. Ando-like maps for $M, N \geq 3$}

Let us now consider the case $k=-1$ from the Section IV B, which can be easily generalized to the $\mathbb{C}^{M} \otimes \mathbb{C}^{N}$ case. Namely, for $\rho \in \mathcal{B}\left(\mathbb{C}^{M} \otimes \mathbb{C}^{N}\right)$, we define

$$
\epsilon(X):=\sum_{i=0, j=0}^{M-1, N-1}|i, j\rangle\langle i, j|X| i, j\rangle\langle i, j|,
$$

$$
\Lambda^{M \times N}(\rho):=(M-1) N \epsilon(\rho)+\sum_{m=0}^{N-1} \epsilon\left(S_{B}^{m} \rho S_{B}^{m \dagger}\right)+\alpha \rho,
$$

where $S_{B}$ is the unitary shift operator in the Bob space.

In this subsection we consider first the case of qutrits $(M=N=3)$ and prove the following result.

Theorem 23. The map

$$
\Lambda^{3 \times 3}(\rho)=6 \epsilon(\rho)+\sum_{m=0}^{2} \epsilon\left(S_{B}^{m} \rho S_{B}^{m^{\dagger}}\right)+\alpha \rho
$$

is separable for $|\alpha| \leq 1 / 2$.

Proof. We consider $|\psi\rangle=\sum_{j=0}^{2}\left(\lambda_{0 j}|0 j\rangle+\lambda_{1 j}|1 j\rangle+\lambda_{2 j}|2 j\rangle\right.$, with $N_{i}=\sum_{j=0}^{2}\left|\lambda_{i j}\right|^{2}$ for $i=0,1,2$, and $\sum_{i=0}^{2} N_{i}=1$. We obtain

$$
\begin{aligned}
\Lambda^{3 \times 3}(\rho)= & {\left[\begin{array}{ccc|ccc|ccc}
6\left|\lambda_{00}\right|^{2}+N_{0} & 0 & 0 & \alpha \lambda_{00} \lambda_{10} & \alpha \lambda_{00} \lambda_{11} & \alpha \lambda_{00} \lambda_{12} & \alpha \lambda_{00} \lambda_{20} & \alpha \lambda_{00} \lambda_{21} & \alpha \lambda_{00} \lambda_{22} \\
0 & 6\left|\lambda_{01}\right|^{2}+N_{0} & 0 & \ldots & \ldots & \ldots & \ldots & \ldots & \alpha \lambda_{01} \lambda_{22} \\
0 & 0 & 6\left|\lambda_{02}\right|^{2}+N_{0} & \ldots & \ldots & \ldots & \ldots & \ldots & \alpha \lambda_{01} \lambda_{22} \\
\hline \alpha \lambda_{00}^{*} \lambda_{10}^{*} & \ldots & \ldots & 6\left|\lambda_{10}\right|^{2}+N_{1} & 0 & 0 & \ldots & \ldots & \alpha \lambda_{10} \lambda_{22} \\
\alpha \lambda_{00}^{*} \lambda_{11}^{*} & \ldots & \ldots & 0 & 6\left|\lambda_{11}\right|^{2}+N_{1} & 0 & \ldots & \ldots & \alpha \lambda_{11} \lambda_{22} \\
\alpha \lambda_{00}^{*} \lambda_{12}^{*} & \ldots & \ldots & 0 & 0 & 6\left|\lambda_{12}\right|^{2}+N_{1} & \ldots & \ldots & \alpha \lambda_{12} \lambda_{22} \\
\hline \alpha \lambda_{00}^{*} \lambda_{20}^{*} & \ldots & \ldots & \ldots & \ldots & \ldots & 6\left|\lambda_{20}\right|^{2}+N_{2} & 0 & 0 \\
\alpha \lambda_{01}^{*} \lambda_{21}^{*} & \ldots & \ldots & \ldots & \ldots & \ldots & 0 & 6\left|\lambda_{21}\right|^{2}+N_{2} & 0 \\
\alpha \lambda_{00}^{*} \lambda_{22}^{*} & \alpha \lambda_{10}^{*} \lambda_{22}^{*} & \alpha \lambda_{02}^{*} \lambda_{22}^{*} & \alpha \lambda_{10}^{*} \lambda_{22}^{*} & \alpha \lambda_{11}^{*} \lambda_{22}^{*} & \alpha \lambda_{12}^{*} \lambda_{22}^{*} & \alpha \lambda_{20}^{*} \lambda_{22}^{*} & \alpha \lambda_{21}^{*} \lambda_{22}^{*} & 6\left|\lambda_{22}\right|^{2}+N_{2}
\end{array}\right] . } \\
& +\sum_{i=0}^{2}|i\rangle\left\langle i|\otimes| f_{i}\right\rangle\left\langle f_{i}\right|,
\end{aligned}
$$

where $\left|f_{i}\right\rangle=\sum_{j=0}^{2} \lambda_{i j}|j\rangle$. The proof follows exactly the same steps as in the previous section: we subsequently get rid of coherences in the above matrix by substracting matrices that live in $2 \times 2$ space and are explicitely separable, provided $|\alpha| \leq 1$. As before we end up with the conditions:

$$
\left[\begin{array}{ccc}
(6-4|\alpha|)\left|\lambda_{i 0}\right|^{2}+(1-2|\alpha|) N_{i} & 0 & 0 \\
0 & (6-4|\alpha|)\left|\lambda_{i 1}\right|^{2}+(1-2|\alpha|) N_{i} & 0 \\
0 & 0 & (6-4|\alpha|)\left|\lambda_{i 2}\right|^{2}+(1-2|\alpha|) N_{i}
\end{array}\right]+\alpha\left|f_{i}\right\rangle\left\langle f_{i}\right| \geq 0
$$

Clearly, we must have $|\alpha| \leq 1 / 2$ to assure non-negativity.

Moreover, using again Ref. [34] we obtain that

$$
\sum_{j=0}^{2} \frac{|\alpha|\left|\lambda_{0 j}\right|^{2}}{(6-4|\alpha|)\left|\lambda_{0 j}\right|^{2}+(1-2|\alpha|) N_{0}} \leq 1
$$


which implies $-9 / 13 \leq \alpha$, so that ultimately the condition $|\alpha| \leq 1 / 2$ is decisive.

Generalization to the case of arbitrary $M \times N$ yields

$$
\sum_{j=0}^{N-1} \frac{|\alpha|\left|\lambda_{0 j}\right|^{2}}{(M-1)(N-2|\alpha|)\left|\lambda_{0 j}\right|^{2}+(1-(M-1)|\alpha|) N_{0}} \leq 1,
$$

which leads to $-N M /(N M+2(M-1) \leq \alpha$, which is also less restrictive that $|\alpha| \leq 1 / 2$, i.e. irrelevant. Therefore we have the following general result.

Theorem 24. If $\rho \geq 0$, then

$$
\Lambda^{M \times N}(\rho)=(M-1) N \epsilon(\rho)+\sum_{m=0}^{N-1} \epsilon\left(S_{B}^{m} \rho S_{B}^{m \dagger}\right)+\alpha \rho
$$

is separable for $|\alpha| \leq 1 / 2$.

Similar results can be proven analogously for the $k$ dependent $\Lambda$, namely, for $k=0,1, \ldots$, we consider the following map

$$
\begin{aligned}
\Lambda_{k}^{M \times N}(\rho) & :=(N-1) \epsilon(\rho)+\sum_{i=0}^{M-2} \sum_{j=0}^{N-1} \epsilon\left(S_{A}^{i} S_{B}^{j} \rho S_{A}^{i \dagger} S_{B}^{j \dagger}\right) \\
& +\sum_{j=0}^{k} \epsilon\left(S_{A}^{M-1} S_{B}^{j} \rho S_{A}^{M-1 \dagger} S_{B}^{j \dagger}\right)+\alpha \rho .
\end{aligned}
$$

Theorem 25. If $\rho \geq 0$, then $\Lambda_{0}^{M \times N}(\rho)$, as defined in Eq. (29), is separable for $\max [-(2 N-1) /(N+(N+2)(M-1)),-1 / 2] \leq$ $\alpha \leq 1 / 2$.

Proof. As above we first present the proof for $M=N=3$ in more detail. The starting point is to write down the explicit matrix from of $\Lambda^{3 \times 3}(\rho)$. Performing the same steps as before and assuming that $|\alpha| \leq 1$ we end up with the condition for separability

$$
\begin{aligned}
\operatorname{diag} & \left\{(2-4|\alpha|)\left|\lambda_{00}\right|^{2}+(1-2|\alpha|) N_{0}+N_{1}+\left|\lambda_{20}\right|^{2},\right. \\
& (2-4|\alpha|)\left|\lambda_{01}\right|^{2}+(1-2|\alpha|) N_{0}+N_{1}+\left|\lambda_{21}\right|^{2}, \\
& \left.(2-4|\alpha|)\left|\lambda_{02}\right|^{2}+(1-2|\alpha|) N_{0}+N_{1}+\left|\lambda_{22}\right|^{2}\right\} \\
& +\alpha\left|f_{i}\right\rangle\left\langle f_{i}\right| \geq 0
\end{aligned}
$$

and analogous conditions for $i=1,2$. Clearly, $|\alpha| \leq 1 / 2$ is necessary; a conservative bound gives $-5 / 13 \leq \alpha$. Generalization to arbitrary $M, N$ leads to

$$
\sum_{j=0}^{N-1} \frac{|\alpha|\left|\lambda_{0 j}\right|^{2}}{(N-1-2(M-1)|\alpha|)\left|\lambda_{0 j}\right|^{2}+(1-(M-1)|\alpha|) N_{0}} \leq 1,
$$

implying $\max [-(2 N-1) /(N+(N+2)(M-1)),-1 / 2] \leq \alpha$.

\section{Examples}

Similar to Section IV C, we will present some examples of states which lie outside the separable ball, but nonetheless are detected to be separable by our simplest criterion in Eq. (27).

Let us consider the following class of $3 \otimes 3$ states from Ref. [39],

$$
\rho_{\beta}=\frac{2}{7}|\Phi\rangle\langle\Phi|+\frac{\beta}{7} \sigma_{+}+\frac{5-\beta}{7} \sigma_{-}, \quad \beta \in[0,5],
$$

where $|\Phi\rangle=\frac{1}{\sqrt{3}} \sum_{k=0}^{2}|k k\rangle$,

$$
\begin{aligned}
& \sigma_{+}=\frac{1}{3}(|01\rangle\langle 01|+| 12\rangle\langle 12|+| 20\rangle\langle 20|), \\
& \sigma_{-}=\frac{1}{3}(|10\rangle\langle 10|+| 21\rangle\langle 21|+| 02\rangle\langle 02|) .
\end{aligned}
$$

This is an interesting class of states: for $0 \leq \beta<1$ and $4<\beta \leq 5$ it is NPT, for $2 \leq \beta \leq 3$ it is separable, and for $3<\beta \leq 4$ it is bound entangled. One verifies that for $0 \leq \beta<(110-\sqrt{4495}) / 44$, or $(110+\sqrt{4495}) / 44<\beta \leq 5$, $\rho_{\beta}$ is NPT (hence entangled), but for all $\alpha \in[-1 / 2,1 / 2]$, $\Lambda^{3 \times 3}\left(\rho_{\beta}\right)$ lies outside the separable ball yet detected to be separable by Eq. (27). Similar examples can be constructed for the other maps as well.

\section{CONCLUSIONS AND OUTLOOK}

We have presented in this paper several families of linear maps, that have a property that when applied to states, result in separable states, or states from a certain class of entanglement. When invertible, such maps allow to derive sufficient crtiteria for separability, or for a certain class of entanglement. Such criteria have rather obvious possible applications and implications in quantum infomation science: they can namely be applied to determine whether and how much entanglement is needed for various specific protocols for quantum computation or other quantum task, similarly as it was discussed in Ref. [23]. We have formulated also several open questions in particular concerning certain kind of BreuerHall-like and reduction-like maps acting in $\mathcal{B}\left(\mathbb{C}^{M} \otimes \mathbb{C}^{N}\right)$, Ando-like maps acting in $\mathcal{B}\left(\mathbb{C}^{M} \otimes \mathbb{C}^{N}\right)$, and certain noninvertible maps in $\mathcal{B}\left(\mathbb{C}^{2} \otimes \mathbb{C}^{N}\right)$ and $\mathcal{B}\left(\mathbb{C}^{4} \otimes \mathbb{C}^{N}\right)$. It is quite remarkable that even our simplest criteria obtained for the case of reduction-like maps is independent to some well-known criteria from the literature. We have also presented different proof for already known results on separability of some class of states, and hopefully the technique is applicable for other, so far not-consideredin-literature situations, such as the case of many parties, symmetric states etc. 


\section{ACKNOWLEDGMENTS}

ML, RA, and SR acknowledge financial support from the John Templeton Foundation, the EU grants OSYRIS (ERC-2013-AdG Grant No. 339106), QUIC (H2020-
FETPROACT-2014 No. 641122), and SIQS (FP7-ICT2011-9 No. 600645), the Spanish MINECO grants FOQUS (FIS2013- 46768-P) and "Severo Ochoa" Programme (SEV-2015-0522), and the Generalitat de Catalunya grant 2014 SGR 874.
[1] R. Horodecki, P. Horodecki, M. Horodecki, and K. Horodecki, Rev. Mod. Phys. 81, 865 (2009).

[2] L. Gurvits, J. Comput. System Sci. 69, 448 (2003).

[3] A. C. Doherty, P. A. Parrilo, and F. M. Spedalieri, Phys. Rev. Lett. 88, 187904 (2002).

[4] A. Peres, Phys. Rev. Lett. 77, 1413 (1996).

[5] M. Horodecki, P. Horodecki, and R. Horodecki, Phys. Lett. A 223, 1 (1996).

[6] M. Horodecki and P. Horodecki, Phys. Rev. A 59, 4206 (1999).

[7] N. J. Cerf, C. Adami, and R. M. Gingrich, Phys. Rev. A 60, 898 (1999).

[8] H.-P. Breuer, Phys. Rev. Lett. 97, 080501 (2006).

[9] W. Hall, J. Phys. A: Math. Gen. 39, 14119 (2006).

[10] M.-D. Choi, Linear Algebr. Appl. 10, 285 (1975).

[11] J. Fiurasek, Phys. Rev. A 66, 052315 (2002).

[12] P. Horodecki and A. Ekert, Phys. Rev. Lett. 89, 127902 (2002); P. Horodecki, Phys. Rev. A 68, 052101 (2003).

[13] J. K. Korbicz, M. L. Almeida, J. Bae, M. Lewenstein and A. Acin, Phys. Rev. A 78, 062105 (2008).

[14] R. Augusiak, J. Bae, Ł. Czekaj, and M. Lewenstein, J. Phys. A: Math. Theor. 44, 185308 (2011).

[15] D. Chruściński and G. Sarbicki, J. Phys. A: Math. Theor. 47, 195301 (2014).

[16] H. A. Carteret, Phys. Rev. Lett. 94, 040502 (2005).

[17] P. Horodecki, R. Augusiak, M. Demianowicz, Phys. Rev. A 74, 052323 (2006).

[18] M. Lewenstein, B. Kraus, J. I. Cirac, and P. Horodecki, Phys. Rev. A 62, 052310 (2000).

[19] M. Lewenstein, B. Kraus, P. Horodecki, and J. I. Cirac, Phys. Rev. A 63, 044304 (2001).

[20] O. Gühne, P. Hyllus, D. Bruß, A. Ekert, M. Lewenstein, C. Macchiavello, and A. Sanpera, J. Mod. Opt. 50, 1079,
(2003).

[21] G. Vidal and R. Tarrach, Phys. Rev. A 59, 141 (1999).

[22] K. Życzkowski, P. Horodecki, A. Sanpera, and M. Lewenstein, Phys. Rev. A 58, 883 (1998).

[23] S. L. Braunstein, C. M. Caves, R. Jozsa, N. Linden, S. Popescu, and R. Schack, Phys. Rev. Lett. 83, 1054 (1999).

[24] L. Gurvits and H. Barnum, Phys. Rev. A 66, 062311 (2002).

[25] S. J. Szarek, Phys. Rev. A 72, 032304 (2005).

[26] G. Aubrun and S. J. Szarek, Phys. Rev. A 73, 022109 (2006).

[27] A. Peres, Quantum Theory: Concepts and Methods, ISBN: 978-0-7923-3632-7, Springer Netherlands (1995).

[28] B. M. Terhal and P. Horodecki, Phys. Rev. A 61, 040301(R) (2000).

[29] A. Sanpera, D. Bruß, and Maciej Lewenstein, Phys. Rev. A 63, 050301(R) (2001).

[30] B. Kraus, J. I. Cirac, S. Karnas, and M. Lewenstein, Phys. Rev. A 61, 062302 (2000).

[31] K. Tanahasi and J. Tomiyama, Can. Math. Bull. 31, 308 (1988).

[32] H. Osaka, Linear Algebr. Appl. 153, 73 (1991); ibid. 186, 45 (1993).

[33] K.-C. Ha, Publ. Res. Inst. Math. Sci. 34, 591 (1998).

[34] M. Lewenstein and A. Sanpera, Phys. Rev. Lett. 80, 2261 (1998).

[35] P. Horodecki, Phys. Lett. A 232, 333 (1997).

[36] D. Chruściński, J. Pytel, and G. Sarbicki, Phys. Rev. A 80, 062314 (2009).

[37] D. Chruściński and J. Pytel, Phys. Rev. A 82, 052310 (2010).

[38] L. Clarisse, J. Phys. A: Math. Gen. 39, 4239 (2006).

[39] M. Horodecki, P. Horodecki, and R. Horodecki, "Mixedstate entanglement and quantum communication", in Quantum Information, G. Albert et al. (Ed.), Springer Tracts in Modern Physics, vol. 173, Springer Verlag Berlin, 2001, p. 151. 